Published on Web 04/07/2006

\title{
A New Reverse Wormlike Micellar System: Mixtures of Bile Salt and Lecithin in Organic Liquids
}

\author{
Shih-Huang Tung, Yi-En Huang, and Srinivasa R. Raghavan* \\ Contribution from the Department of Chemical and Biomolecular Engineering, \\ University of Maryland, College Park, Maryland 20742-2111
}

Received December 9, 2005; Revised Manuscript Received March 14, 2006; E-mail: sraghava@eng.umd.edu

\begin{abstract}
We report a new route for forming reverse wormlike micelles (i.e., long, flexible micellar chains) in nonpolar organic liquids such as cyclohexane and $n$-decane. This route involves the addition of a bile salt (e.g., sodium deoxycholate) in trace amounts to solutions of the phospholipid lecithin. Previous recipes for reverse wormlike micelles have usually required the addition of water to induce reverse micellar growth; here, we show that bile salts, due to their unique "facially amphiphilic" structure, can play a role analogous to that of water and promote the longitudinal aggregation of lecithin molecules into reverse micellar chains. The formation of transient entangled networks of these reverse micelles transforms low-viscosity lecithin organosols into strongly viscoelastic fluids. The zero-shear viscosity increases by more than 5 orders of magnitude, and it is the molar ratio of bile salt to lecithin that controls the viscosity enhancement. The growth of reverse wormlike micelles is also confirmed by small-angle neutron scattering (SANS) experiments on these fluids.
\end{abstract}

\section{Introduction}

The self-assembly of reverse wormlike micelles in organic liquids ("oils") has been studied for more than two decades, following the work of Luisi and co-workers. ${ }^{1-6}$ These micelles are inverted cylindrical chains that grow to lengths of several microns while also maintaining a local flexibility. Because of their flexibility, the micelles have been referred to as wormlike, threadlike, or polymer-like structures. The first examples of such micellar systems were ternary mixtures of the type lecithin/ water/oil. ${ }^{1}$ Lecithin is a zwitterionic phospholipid with two alkyl tails (Figure 1a), which when added alone to oil gives rise to reverse spherical or ellipsoidal micelles. When a small quantity of water is added to these fluids, the micelles grow axially into flexible cylinders. Thus, the crucial component is water, and the molar ratio of water to lecithin (denoted by $w_{0}$ ) is the key parameter in dictating reverse micellar growth. The growth of these micellar chains and their subsequent entanglement into a transient network transforms the sample into a highly viscous and viscoelastic one. ${ }^{2,6}$ In turn, the viscosity in the limit of low shear rates, i.e., the zero-shear viscosity $\eta_{0}$, is enhanced by several orders of magnitude relative to that of neat lecithin solutions.

Wormlike micellar structures in water have also garnered a great deal of attention over the past two decades. ${ }^{7-9}$ Such micelles can be formed in water by a variety of surfactants,

(1) Scartazzini, R.; Luisi, P. L. J. Phys. Chem. 1988, 92, 829-833.

(2) Schurtenberger, P.; Scartazzini, R.; Luisi, P. L. Rheol. Acta 1989, 28, 372381.

(3) Luisi, P. L.; Scartazzini, R.; Haering, G.; Schurtenberger, P. Colloid Polym. Sci. 1990, 268, 356-374.

(4) Schurtenberger, P.; Scartazzini, R.; Magid, L. J.; Leser, M. E.; Luisi, P. L. J. Phys. Chem. 1990, 94, 3695-3701.

(5) Schurtenberger, P.; Magid, L. J.; King, S. M.; Lindner, P. J. Phys. Chem. 1991, 95, 4173-4176.

(6) Shchipunov, Y. A. Colloids Surf., A 2001, 183, 541-554.

10.1021/ja0583766 CCC: $\$ 33.50$ @ 2006 American Chemical Society

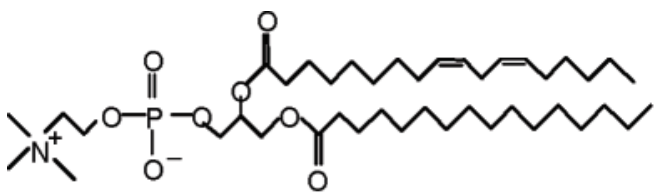

(a)

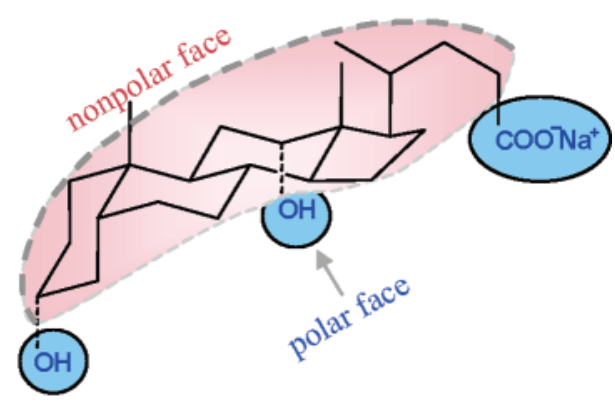

(b)

Figure 1. Molecular structures of (a) soybean lecithin and (b) the bile salt sodium deoxycholate (SDC). The facially amphiphilic structure of the bile salt is highlighted in the schematic.

including cationic, anionic, nonionic, and zwitterionic. ${ }^{8}$ In the case of ionic surfactants, wormlike micelles are typically induced by adding salt to the solution. The added salt screens the ionic repulsions between the surfactant headgroups and thereby promotes the growth of micelles. In the case of cationic surfactants, which have been studied the most, both simple

(7) Cates, M. E.; Candau, S. J. J. Phys.: Condens. Matter 1990, 2, 68696892.

(8) Hoffmann, H. In Structure and Flow in Surfactant Solutions; Herb, C. A. Prud'homme, R. K., Eds.; American Chemical Society: Washington, DC, 1994; pp 2-31.

(9) Raghavan, S. R.; Kaler, E. W. Langmuir 2001, 17, 300-306. 
electrolytes (e.g., sodium chloride) as well as salts with aromatic counterions (e.g., sodium salicylate, NaSal) can induce the growth of worms. ${ }^{8,9}$ The aromatic salts tend to be especially effective because their counterions bind to the surface of the micelles and thereby reduce the surface charge. As a result, these aromatic salts can promote cationic micellar growth at very low concentrations, i.e., at salt/surfactant molar ratios much less than one. Similar aromatic binding salts capable of inducing anionic wormlike micelles have also been demonstrated recently. ${ }^{10}$

Interest in wormlike micelles continues to grow, both from industry (e.g., in oilfield applications) ${ }^{11}$ and also from soft matter theorists. ${ }^{7,12}$ It is now widely recognized that these micelles are very similar to polymeric chains with the important exception that the micelles are in thermal equilibrium with their monomers. ${ }^{7}$ In other words, the micelles break and recombine at a rapid rate, and a competition between micellar breaking and chain reptation dictates the rheology of the fluid. If the breaking time $\tau_{\mathrm{B}}$ is much lower than the reptation time $\tau_{\text {rep }}$, the sample rheology becomes very simple and well-defined, with just a single relaxation time whose value is the geometric mean of $\tau_{\mathrm{B}}$ and $\tau_{\text {rep. }}{ }^{7}$ The sample can then be described as a singlerelaxation-time Maxwell fluid. Both normal and reverse worms can show Maxwell fluidlike behavior. ${ }^{6,8}$

Returning now to reverse worms, it is surprising that their formation has been reported in only a few systems to-date. ${ }^{13-16}$ The lecithin/water/oil system continues to be the system of choice for most studies dealing with reverse worms. Recent studies have focused on the role played by water in reverse micellar growth (water can be substituted with other polar solvents such as glycerol). ${ }^{17-22}$ These studies have yielded disparate and sometimes diverging conclusions; some have speculated that water is a necessary "glue" that holds these reverse micelles together, but this has been refuted by others. However, it is generally accepted that the formation of lecithin reverse worms requires the addition of water or an analogous polar solvent.

In this paper, we report that an entirely different class of additives, bile salts, can induce lecithin to form reverse worms in organic solvents. Bile salts are physiological surfactants that play an important role in our body, such as in the digestion of fat and the excretion of excess cholesterol. ${ }^{23,24}$ Structurally, bile salts are an unusual class of amphiphiles: unlike typical surfactants, which present a polar head and a nonpolar tail, bile salts are facial amphiphiles, with a polar and a nonpolar face (Figure 1b). ${ }^{23}$ We will propose that this "facially amphiphilic" structure of bile salts is critical to their ability to induce the

(10) Hassan, P. A.; Raghavan, S. R.; Kaler, E. W. Langmuir 2002, 18, 25432548 .

(11) Yang, J. Curr. Opin. Colloid Interface Sci. 2002, 7, 276-281.

(12) Cates, M. E. Macromolecules 1987, 20, 2289-2296.

(13) Harrison, W. J.; McDonald, M. P.; Tiddy, G. J. T. J. Phys. Chem. 1991 95, 4136-4140.

(14) Yu, Z. J.; Neuman, R. D. Langmuir 1994, 10, 2553-2558.

(15) Steytler, D. C.; Jenta, T. R.; Robinson, B. H.; Eastoe, J.; Heenan, R. K. Langmuir 1996, 12, 1483-1489.

(16) Hellweg, T.; Eimer, W. Colloids Surf., A 1998, 136, 97-107.

(17) Capitani, D.; Rossi, E.; Segre, A. L.; Giustini, M.; Luisi, P. L. Langmuir 1993, 9, 685-689.

(18) Shchipunov, Y. A.; Shumilina, E. V. Mater. Sci. Eng., C 1995, 3, 43-50.

(19) Angelico, R.; Palazzo, G.; Colafemmina, G.; Cirkel, P. A.; Giustini, M.; Ceglie, A. J. Phys. Chem. B 1998, 102, 2883-2889.

(20) Willard, D. M.; Riter, R. E.; Levinger, N. E. J. Am. Chem. Soc. 1998, 120, $4151-4160$

(21) Aliotta, F.; Fontanella, M. E.; Lechner, R. E.; Pieruccini, M.; Ruffle, B.; Vasi, C. Phys. Rev. E 1999, 60, 7131-7136.

(22) Ibrahim, T. H.; Neuman, R. D. Langmuir 2004, 20, 3114-3122.

(23) Hofmann, A. F.; Small, D. M. Annu. Rev. Med. 1967, 18, 333.

(24) Carey, M. C.; Small, D. M. Am. J. Med. 1970, 49, 590. growth of lecithin reverse worms; indeed, the role of bile salt is potentially quite analogous to that of water in this regard. Interestingly also, we find that micellar growth is induced by very small concentrations of bile salt, i.e., molar ratios of bile salt to lecithin much less than one. In this regard, the action of bile salts on reverse micelles is similar to the action of aromatic salts such as NaSal on cationic micelles in water. This similarity implies a strong binding of bile salts with the reverse micelles, and we will elaborate on this point later in the paper.

Apart from their scientific peculiarity, there are other reasons for studying bile salt-lecithin reverse micelles. Currently, there is much interest in using reverse micelles as hosts for enzymes $^{25-27}$ and also in drug delivery. ${ }^{28-30}$ Studies have found that biomolecules or drugs encapsulated in reverse micelles can show good biological activity; however, the stability of these molecules is often inversely related to the amount of water present in the formulation. The reverse micelles described here are formed without any added water and could thereby offer some advantages for the encapsulation of biological or organic molecules. In addition, the study of these reverse micelles could also provide insights into physiological processes involving lecithin, bile salt, and nonpolar substances such as fats or fatty acids.

\section{Results}

Lecithin/SDC/Cyclohexane: Phase Behavior and Rheology. We first focus on mixtures of lecithin and the bile salt sodium deoxycholate (SDC) in cyclohexane. Results with other bile salts and in other nonpolar solvents will be discussed later. We chose cyclohexane because our results could be compared with those for lecithin/water/cyclohexane mixtures from the literature. In cyclohexane, lecithin forms reverse spherical or ellipsoidal micelles, and the resulting solutions have a low viscosity, essentially identical to that of the solvent. ${ }^{6}$ Adding SDC to these solutions increases the viscosity dramatically. This is evident even by visual observation; for example, the sample barely flows when the vial is tilted, and bubbles remain trapped in the fluid for long periods of time. To quantify the effect of SDC, we conducted rheological experiments at a fixed lecithin concentration of $100 \mathrm{mM}$ and with increasing concentrations of SDC. The results are expressed as a function of $B_{0}$, the molar ratio of bile salt to lecithin.

Figure 2 shows the zero-shear viscosity $\eta_{0}$ of $100 \mathrm{mM}$ lecithin solutions as a function of $B_{0}$. The values of $\eta_{0}$ were obtained from steady-shear rheological experiments in the limit of low shear rates, where the viscosity asymptotically approached a plateau. We note that $\eta_{0}$ increases by 5 orders of magnitude as $B_{0}$ increases from 0.2 to 0.45 . All these solutions are transparent and isotropic at rest, with the more viscous samples showing a weak birefringence under flow (e.g., on shaking a vial). Photographs of sample vials for $B_{0}=0.2$ and 0.4 are also shown in Figure 2. The $B_{0}=0.4$ sample is shown in an inverted vial to highlight how slowly the sample flows under gravity because of its high viscosity. Note that, because this sample does flow,

(25) Knezevic, Z. D.; Siler-Marinkovic, S. S.; Mojovic, L. V. Appl. Microbiol. Biotechnol. 1998, 49, 267-271.

(26) Madamwar, D.; Thakar, A. Appl. Biochem. Biotechnol. 2004, 118, 361369.

(27) Van Horn, W. D.; Simorellis, A. K.; Flynn, P. F. J. Am. Chem. Soc. 2005 , 127, 13553-13560.

(28) Dreher, F.; Walde, P.; Walther, P.; Wehrli, E. J. Controlled Release 1997 $45,131-140$.

(29) Kreilgaard, M. Adv. Drug Delivery Rev. 2002, 54, S77-S98.

(30) Kumar, R.; Katare, O. P. AAPS PharmSciTech 2005, 6. 


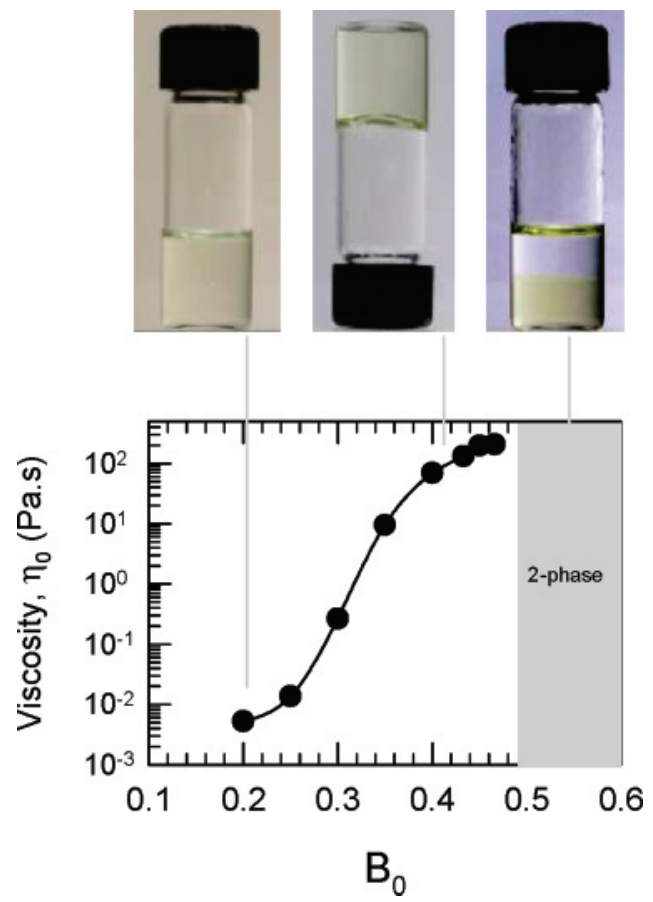

Figure 2. Zero-shear viscosity $\eta_{0}$ of lecithin + bile salt (SDC) in cyclohexane at $25{ }^{\circ} \mathrm{C}$ as a function of $B_{0}$, the molar ratio of bile salt to lecithin, with the lecithin concentration held constant at $100 \mathrm{mM}$. Photographs of three samples corresponding to different $B_{0}$ values are also shown. At low $B_{0}$, the sample is a solution of low viscosity. At a $B_{0}$ around 0.4 , the sample viscosity is a factor of $10^{5}$ higher and the sample flows very slowly in the overturned vial. Finally, when $B_{0}$ exceeds ca. 0.5 , the sample phase-separates into two coexisting liquid phases.

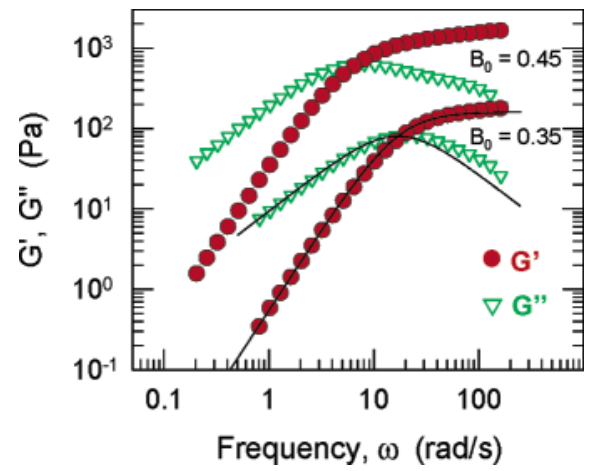

Figure 3. Dynamic rheology at $25^{\circ} \mathrm{C}$ of two lecithin-SDC mixtures in cyclohexane. The samples contain $100 \mathrm{mM}$ lecithin and the SDC/lecithin molar ratios, $B_{0}=0.35$ and 0.45 . The plot shows the elastic modulus $G^{\prime}$ $(\bullet)$ and the viscous modulus $G^{\prime \prime}(\nabla)$ as functions of frequency $\omega$. Fits to a single-relaxation-time Maxwell model are shown as solid lines for the $B_{0}$ $=0.35$ sample.

albeit slowly, it cannot be termed a "gel"; the dynamic rheological data (Figure 3) confirm this point. A further interesting observation is that, above a $B_{0}$ of 0.45 , lecithin-SDC samples in cyclohexane phase-separate into two isotropic liquid phases, one nonviscous and the other viscous (see photograph of typical sample on the top right in Figure 2). The lower phase, which is similar in viscosity to the one-phase samples at lower $B_{0}$, contains most of the lecithin and SDC. The upper, nonviscous phase is a very dilute reverse micellar solution. A similar phase separation occurs for the lecithin-water-cyclohexane system at higher water-lecithin molar ratios. ${ }^{6}$

To characterize the viscoelasticity of lecithin-SDC reverse worms, we turned to dynamic rheology. Figure 3 shows representative dynamic rheological data (elastic modulus $G^{\prime}$ and

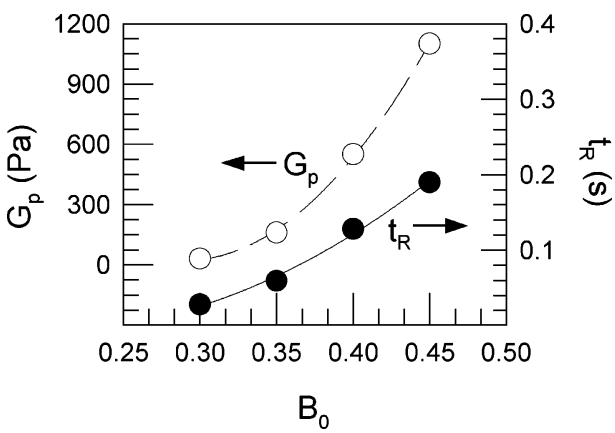

Figure 4. Plateau modulus $G_{\mathrm{p}}$ and relaxation time $t_{\mathrm{R}}$ for lecithin-SDC mixtures in cyclohexane as a function of the SDC/lecithin molar ratio $B_{0}$. The lecithin concentration in these samples is $100 \mathrm{mM}$. The parameters were extracted from dynamic rheological spectra such as those shown in Figure 3.

the viscous modulus $G^{\prime \prime}$ as functions of frequency $\omega$ ) for two samples containing $100 \mathrm{mM}$ lecithin with $B_{0}=0.35$ and 0.45 . The data clearly reveal the viscoelastic response of these samples. That is, at high $\omega$ or short time scales, the samples show elastic behavior, with $G^{\prime}$ tending to a plateau and dominating over $G^{\prime \prime}$. On the other hand, at low $\omega$ or long time scales, the samples show viscous behavior, with $G^{\prime \prime}$ exceeding $G^{\prime}$ and the slopes of $G^{\prime}$ and $G^{\prime \prime}$ being close to 2 and 1 , respectively, on the $\log -\log$ plot. The dominant relaxation time $t_{\mathrm{R}}$ of these viscoelastic samples can be estimated as $1 / \omega_{\mathrm{c}}$, where $\omega_{\mathrm{c}}$ is the frequency at which $G^{\prime}$ and $G^{\prime \prime}$ cross. In Figure 3, we also show fits to $G^{\prime}(\omega)$ and $G^{\prime \prime}(\omega)$ for the 0.35 sample using a Maxwell model with a single relaxation time. The predictions of this model are ${ }^{7}$

$$
\begin{aligned}
G^{\prime}(\omega) & =\frac{G_{\mathrm{p}} \omega^{2} t_{\mathrm{R}}{ }^{2}}{1+\omega^{2} t_{\mathrm{R}}{ }^{2}} \\
G^{\prime \prime}(\omega) & =\frac{G_{\mathrm{p}} \omega t_{\mathrm{R}}}{1+\omega^{2} t_{\mathrm{R}}{ }^{2}}
\end{aligned}
$$

Here, $G_{\mathrm{p}}$ is the plateau modulus, i.e., the value of $G^{\prime}$ in the high-frequency limit. We note that the Maxwell model fits the data reasonably well, especially at low and intermediate frequencies, while there is a slight discrepancy at high frequencies. This confirms that a single relaxation time (or a narrow spectrum of relaxation times) dominates the rheological response of this sample. Maxwell fluidlike behavior is indicative of entangled wormlike micelles, both normal and reversed. ${ }^{7}$

Figure 4 shows the variation of dynamic rheological parameters with bile salt concentration. Here, the plateau modulus $G_{\mathrm{p}}$ and the relaxation time $t_{\mathrm{R}}\left(=1 / \omega_{\mathrm{c}}\right)$ are plotted as a function of $B_{0}$ for a fixed lecithin concentration of $100 \mathrm{mM}$. Both $G_{\mathrm{p}}$ and $t_{\mathrm{R}}$ are seen to increase steeply with $B_{0}$. The increase of relaxation time $t_{\mathrm{R}}$ with $B_{0}$ is related to the growth of reverse micellar chains upon addition of bile salt. A similar increase in $t_{\mathrm{R}}$ is seen for cationic worms in water as they grow upon addition of aromatic salts. ${ }^{8,9}$ However, the sharp increase in plateau modulus $G_{\mathrm{p}}$ is unexpected. For comparison, the $G_{\mathrm{p}}$ of cationic worms is generally independent of salt for a fixed surfactant content. ${ }^{9}$ An increase in $G_{\mathrm{p}}$ generally implies an increase in the volume fraction of entangled micelles. ${ }^{7}$ One can thereby consider the bile salt to have a dual rule: it not only induces the growth of lecithin reverse micelles but also induces a greater 


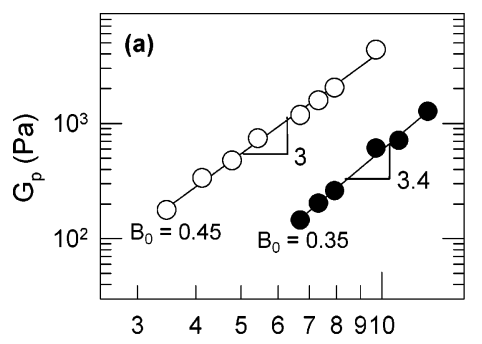

$\phi(\%)$

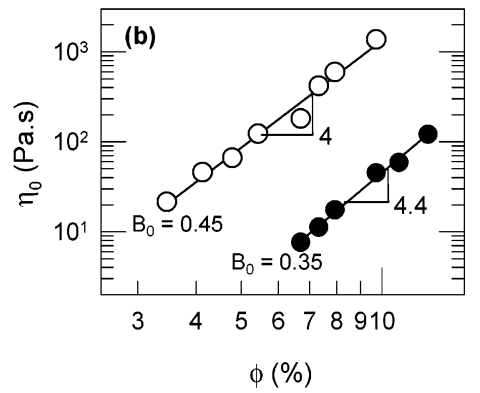

Figure 5. Rheological parameters for lecithin-SDC mixtures as a function of the lecithin volume fraction $\phi$. Data are provided for two values of the SDC/lecithin molar ratio $B_{0}$. Figure 5 a shows the plateau modulus $G_{\mathrm{p}}$, and Figure $5 b$, the zero-shear viscosity $\eta_{0}$.

number of such micelles to form. A mechanism where bile salt molecules are incorporated into the body of the reverse micelle can explain these results and is discussed later in this paper.

We now describe the variation of rheological parameters with lecithin volume fraction $\phi$ at fixed values of the bile salt-tolecithin molar ratio $B_{0}$. The scaling of the plateau modulus $G_{\mathrm{p}}$ vs $\phi$ is shown in Figure 5a for two different $B_{0}$ values $(0.35$ and 0.45 ), and similar data for the zero-shear viscosity $\eta_{0}$ vs $\phi$ are shown in Figure $5 \mathrm{~b}$. As expected, both $G_{\mathrm{p}}$ and $\eta_{0}$ increase steeply with $\phi$, with the data following power laws. However, the power law exponents are unexpectedly large. Taking the case of $G_{\mathrm{p}}$ first, the reptation theory of De Gennes predicts $G_{\mathrm{p}}$ $\approx \phi^{2.25}$ for semidilute entangled polymers. ${ }^{31}$ The same exponent of 2.25 is predicted in the semidilute regime for entangled linear worms as well, and this has been verified experimentally for many aqueous and reverse wormlike micellar systems. ${ }^{7-9}$ Here, on the other hand, the power-law exponents from Figure 5a for $G_{\mathrm{p}}$ are $3.4 \pm 0.2$ for $B_{0}=0.35$ and $3.0 \pm 0.1$ for $B_{0}=0.45$. These values are considerably larger than the theoretical exponent of 2.25, and the discrepancy arises possibly because the bile salt induces an increase in the volume fraction of reverse wormlike micelles. A similar scenario occurs with the $\eta_{0}$ vs $\phi$ data in Figure $4 \mathrm{~b}$, where the power-law exponents are $4.4 \pm$ 0.2 and $4.0 \pm 0.2$ for $B_{0}=0.35$ and $B_{0}=0.45$, respectively. These exponents are larger than those typically reported for nonionic or charge-screened wormlike micelles in water. ${ }^{7-9}$

Lecithin/SDC/Cyclohexane: SANS. Having characterized the rheology, we now turn to SANS to elucidate the underlying microstructure in lecithin-bile salt samples. For these experiments, samples were made in deuterated cyclohexane (these samples were rheologically identical to those made in cyclohexane). We fixed the lecithin concentration at a relatively low value of $20 \mathrm{mM}$ for the SANS experiments so as to keep the micellar volume fraction low and thereby minimize intermicellar

(31) De Gennes, P. G. Scaling Concepts in Polymer Physics; Cornell University Press: Ithaca, NY, 1979.

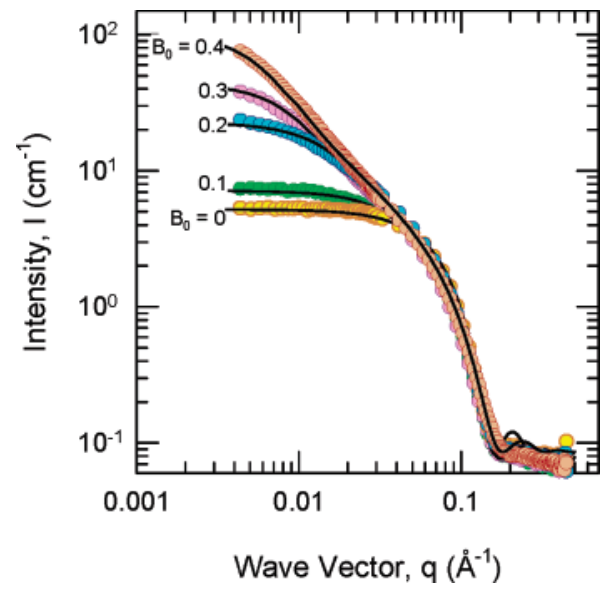

Figure 6. SANS data from samples in deuterated cyclohexane containing $20 \mathrm{mM}$ lecithin and different SDC/lecithin molar ratios $B_{0}$. The solid curves through the data are fits to appropriate models (see text for details).

interactions (structure factor contributions). SANS spectra ( $I$ vs q) for $20 \mathrm{mM}$ lecithin solutions containing varying amounts of bile salt $\left(B_{0}\right.$ ranging from 0 to 0.4$)$ are shown in Figure 6. Clearly, the addition of bile salt causes a dramatic increase in the low- $q$ scattering while the intensity at higher $q$ remains practically unchanged. The increase in low- $q$ scattering is consistent with the growth of elongated structures. ${ }^{32}$ Thus, the SANS data immediately provides a qualitative confirmation of reverse micellar growth induced by the bile salt.

To obtain a more quantitative picture of micellar sizes, we model the SANS data using appropriate form factors, as described in the Supporting Information (eqs S1-S5). The fits are shown as solid curves through the data in Figure 6. First, for the case of lecithin in cyclohexane $\left(B_{0}=0\right)$, the micelles are modeled as ellipsoids of revolution (eq S1) with radii of 22 $\AA$ and $33 \AA$, respectively, for their minor and major axes. From the parameters, these micelles are found to be slightly oblate ellipsoids (incidentally, a model for polydisperse spheres does not fit the data as well). Upon the addition of bile salt, the micelles grow axially. For $B_{0}=0.1$ and 0.2 , the micelles can be modeled as rigid cylinders (eqs S2 and S3). The cylinder radius in each case is about $22 \AA$, while the contour length increases from ca. $96 \AA$ for $B_{0}=0.1$ to ca. $289 \AA$ for $B_{0}=0.2$. With further increase in bile salt content, the micelles become even longer and their flexibility must be taken into account in modeling the SANS data. Thus, for $B_{0}=0.3$ and 0.4 , the micelles are modeled as semiflexible cylindrical chains (eqs $\mathrm{S} 4$ and S5). For these cases, the cylinder radius is around $22 \AA$, the persistence length is ca. $190 \AA$, and the contour length of the chains increases from ca. $700 \AA$ for $B_{0}=0.3$ to ca. $1440 \AA$ for $B_{0}=0.4$. In sum, the SANS data confirm that adding bile salt causes the rapid growth of reverse wormlike micelles. Our analysis also shows that the cross-sectional radius of the mixed lecithin-bile salt micelles is nearly the same as that of pure lecithin micelles. Thus, bile salts induce longitudinal micellar growth without expanding the micellar cross-section.

Lecithin/SDC Micelles: Growth in Different Organic Solvents. We have studied the bile-salt-induced growth of reverse wormlike micelles in a range of organic solvents. In this context, bile salts are very similar to water, and Figure 7 presents a comparison between the two in terms of their worm-

(32) Pedersen, J. S. Adv. Colloid Interface Sci. 1997, 70, 171-210 


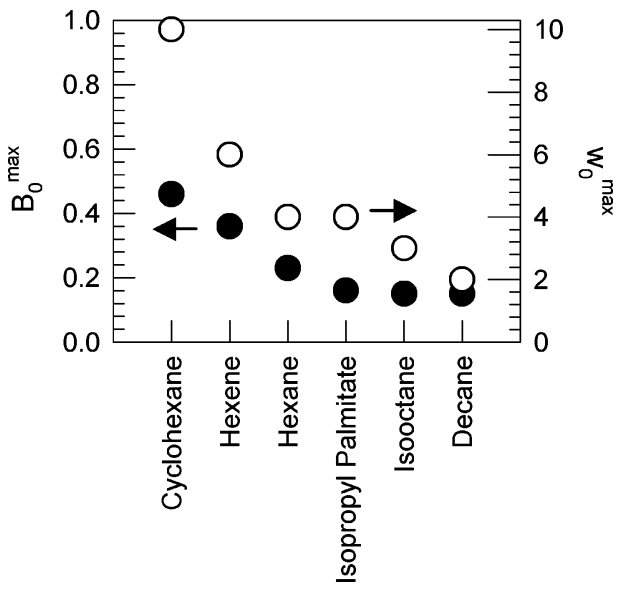

Figure 7. Comparison of bile salt (SDC) and water in terms of their ability to induce viscoelastic reverse micelles of lecithin in six different solvents. The lecithin concentration is fixed at $100 \mathrm{mM}$. The parameters plotted are the molar ratios of bile salt/lecithin $\left(B_{0}{ }^{\max }\right)$ and water/lecithin $\left(w_{0}{ }^{\max }\right)$ at which the zero-shear viscosities of the respective mixtures reach a maximum.

inducing abilities in different solvents. As stated in the Introduction, water is well-known for its ability to induce growth of lecithin reverse worms. Interestingly, when water is added to a lecithin organosol, the viscosity increases to a maximum at a certain water/lecithin molar ratio $w_{0}{ }^{\max }$ while further addition of water typically causes a decrease in viscosity, followed by phase separation. ${ }^{2,6}$ The behavior with bile salts is qualitatively different (Figure 2); the viscosity increases with bile salt content monotonically until the phase boundary (the highest viscosities attained with bile salt and water are quite similar). For comparison, we focus on the molar ratio of bile salt/lecithin at which the viscosity is the highest, i.e., just prior to phase separation, and we denote this value by $B_{0}{ }^{\max }$. Figure 7 thereby compares $B_{0}{ }^{\max }$ (for the bile salt, SDC) and $w_{0}{ }^{\max }$ for six different organic solvents, with the lecithin concentration fixed at $100 \mathrm{mM}$. While the differences from solvent to solvent presumably lie in their interaction with the lecithin headgroup, ${ }^{6}$ the important point from Figure 7 is that $B_{0}{ }^{\max }$ and $w_{0}{ }^{\max }$ generally track each other. For example, $w_{0}{ }^{\max }$ is ca. 10 for cyclohexane and ca. 4 for hexane, while $B_{0}{ }^{\max }$ is 0.45 for cyclohexane and 0.2 for hexane. Note that the $B_{0}{ }^{\max }$ values are much lower than the $w_{0}{ }^{\max }$ values; indeed, very small (less than equimolar) amounts of bile salt are sufficient to substantially increase viscosity. The low values of $B_{0}{ }^{\max }$ imply a strong binding of the bile salt to the lecithin reverse micelle, and this is elaborated further in the Discussion section.

Lecithin Micelles in Cyclohexane: Growth Induced by Different Bile Salts. In addition to SDC, we have studied other bile salts, and a number of these are capable of inducing lecithin to form reverse worms in organic liquids. Figure 8 shows the structures of four such bile salts and a comparison of their worminducing abilities based on their $B_{0}{ }^{\max }$ values in cyclohexane (i.e., the bile salt/lecithin molar ratio at which the viscosity of $100 \mathrm{mM}$ lecithin solutions reaches a maximum). All four bile salts induced highly viscoelastic reverse micellar solutions at less than equimolar ratios relative to the lecithin (the viscosities at the maximum point were comparable). From Figure 8, we note that the $B_{0}{ }^{\max }$ values are quite similar $(\sim 0.4)$ for SDC and sodium cholate (SC), both of which have a carboxylate functionality. The corresponding values for the bile salts with a taurine functionality, i.e., sodium taurodeoxycholate (STDC)
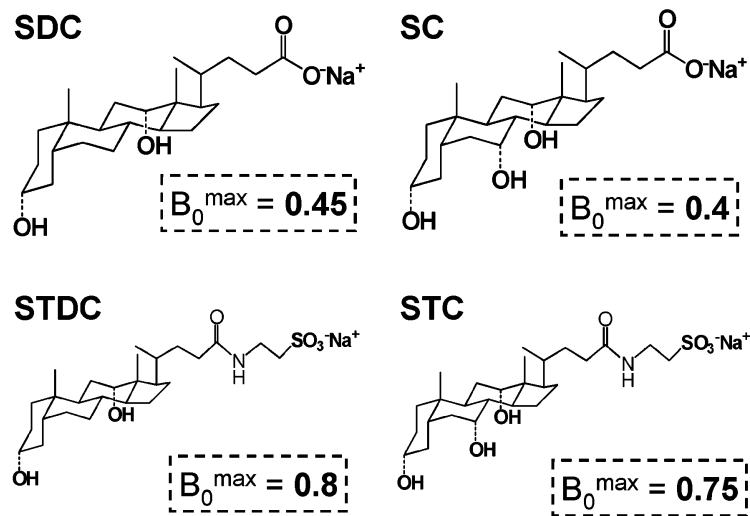

Figure 8. Comparison of four different bile salts in terms of their ability to induce viscoelastic reverse micelles of lecithin in cyclohexane. The lecithin concentration is fixed at $100 \mathrm{mM}$, and $B_{0}{ }^{\max }$ is the molar ratio of the respective bile salt/lecithin mixture at which the zero-shear viscosity reaches a maximum.

and sodium taurocholate (STC), are about twice as high $(\sim 0.8)$, although these values are still lower than equimolar. Based on these results, it appears that the ability to induce growth of reverse micelles is mainly related to the unusual "facially amphiphilic" structure of the bile salt amphiphile. The presence or absence of an extra hydroxyl group and the nature of the headgroup itself are relatively minor factors in terms of the ability of bile salts to induce growth of reverse worms.

\section{Discussion}

In the preceding section, we have shown that bile salts can induce a dramatic growth of lecithin reverse micelles in organic solvents. The presence of long wormlike reverse micelles, in turn, imparts a strong viscoelastic character to the samples. Why are bile salts effective at inducing reverse micellar growth? This is the question that we will address in the current section.

First, it is important to state that the micellar growth is indeed caused by the bile salt and is not an artifact due to residual water in the sample. To underscore this point, we have exhaustively dried both the lecithin and bile salt prior to use, as described in the Experimental Section (see Supporting Information). Still, it is well-known that both soybean lecithin and the bile salts have a residual layer of bound water (ca. equimolar), which cannot be removed by drying. ${ }^{4}$ We have confirmed the presence of residual water at a 0.9:1 molar ratio by ${ }^{1} \mathrm{H}$ NMR studies on dried bile salt samples. However, this residual water content is too small to explain our results. Consider our data for cyclohexane (Figure 7), where $w_{0}{ }^{\max }$, i.e., the water/lecithin molar ratio at the viscosity maximum, is about 10. This means that we must add a significant amount of water to induce a high viscosity. For comparison, the value of $B_{0}{ }^{\max }$, i.e., the bile salt (SDC)/lecithin molar ratio at the viscosity maximum, is 0.45 . Clearly, we require a much lower amount of bile salt to induce a similar viscosity, and the difference is too large to attribute to residual water. Also, as indicated earlier, the progressions in viscosity upon adding water and bile salt are quite different; with water, the viscosity goes through a peak, whereas, with bile salt, the viscosity rises monotonically. Thus, our results clearly show that bile salts have a distinct influence on reverse micellar growth, and we offer below a mechanism to explain their effect. 


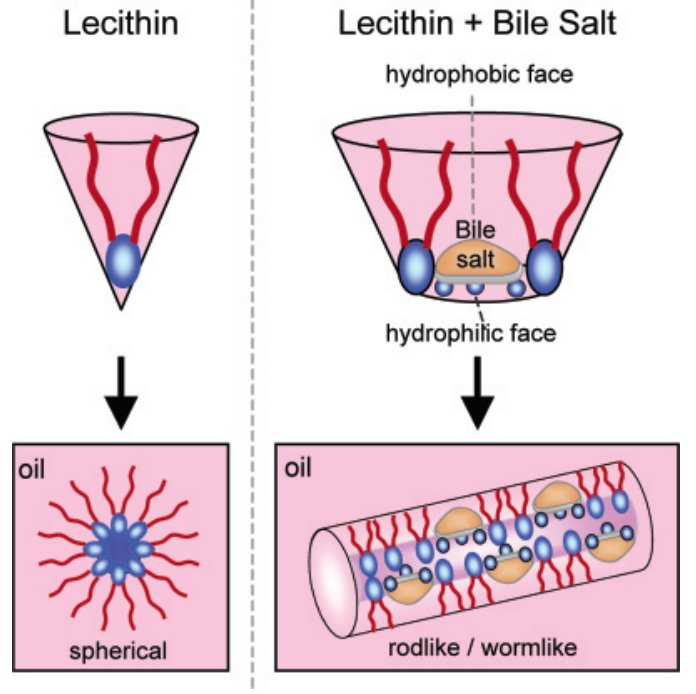

Figure 9. Schematic of the reverse micellar structures formed by lecithin with and without bile salt. Lecithin is shown as a molecule with a blue head and two red tails, while the bile salt is schematically represented following Figure 1b. Lecithin alone tends to form approximately spherical reverse micelles in a nonpolar solvent (oil). When bile salt is added, its hydrophilic moieties bind to the lecithin headgroups, thus expanding the headgroup area. This alters the net geometry from a cone to a truncated cone and thereby induces the spherical micelles to grow into flexible cylinders (worms). Note the orientation of bile salt molecules in the cylindrical micelles; their hydrophilic faces are turned inward, while the hydrophobic faces are exposed to the external oil phase.

The type of self-assembled structure formed by amphiphiles is known to be governed by molecular geometry, and this relationship is usually expressed in terms of the critical packing parameter $p=a_{\text {tail }} / a_{\mathrm{hg}}$ where $a_{\text {tail }}$ and $a_{\mathrm{hg}}$ are the cross-sectional areas of the tail and headgroup, respectively. ${ }^{33}$ In water, ionic surfactants in the absence of salt have a $p$ around $1 / 3$ (i.e., a "cone" shape), which implies the formation of spherical micelles. When salt is added, the effective headgroup area is reduced due to a reduction in the electrostatic screening length. ${ }^{7}$ In turn, the packing parameter $p$ increases to around $1 / 2$ (the molecule adopts a "truncated cone" shape), and the micelles consequently transform from spheres to rods. In the case of organic solvents, the formation of reverse micelles requires a packing parameter $p$ well in excess of 1 , and spherical reverse micelles evidently correspond to an inverse cone shape (Figure 9). For these spheres to grow into rods or worms, the packing parameter $p$ must decrease. We believe that such a decrease is caused in the present lecithin-bile salt system by the binding of bile salt to the lecithin headgroups. ${ }^{34-36}$ In other words, the bile salt increases the headgroup area $a_{\text {hg }}$ while maintaining about the same tail area. The net effect is to decrease $p$ and transform the effective geometry into a truncated cone (Figure 9), thereby driving a transition to long, cylindrical micelles. Note that in the proposed scenario, the bile salt orients its hydrophobic face outward into the organic solvent while its hydrophilic face is directed toward the interior of the micelle (the $-\mathrm{OH}$ groups of the bile salt possibly form hydrogen bonds with the lecithin

(33) Israelachvili, J. Intermolecular and Surface Forces; Academic Press: San Diego, CA, 1991.

(34) Nichols, J. W.; Ozarowski, J. Biochemistry 1990, 29, 4600-4606.

(35) Hjelm, R. P.; Thiyagarajan, P.; Alkanonyuksel, H. J. Phys. Chem. 1992 96, 8653-8661.

(36) Arleth, L.; Bauer, R.; Ogendal, L. H.; Egelhaaf, S. U.; Schurtenberger, P.; Pedersen, J. S. Langmuir 2003, 19, 4096-4104. headgroup). ${ }^{35}$ Thus, we believe the facially amphiphilic structure of the bile salt is the key to its ability to induce lecithin reverse worms.

The above mechanism is supported by previous work on lecithin-bile salt mixtures in water. ${ }^{34-38}$ While lecithin alone tends to form vesicles in water, the addition of bile salt transforms these vesicles into cylindrical micelles. ${ }^{37,38}$ Such a transition implies a reduction in packing parameter from ca. 1 to ca. $1 / 2$. This, in turn, has been attributed to an increase in headgroup area due to the binding of bile salt with lecithin headgroups. We hypothesize a similar binding of bile salt to lecithin in the context of our reverse micellar system.

Finally, we should point out that bile salts appear to have an analogous effect as water in inducing the formation of lecithin reverse worms. A number of studies have suggested that water is distributed in the headgroup region of lecithin reverse micelles and that water molecules form hydrogen bonds with the phosphate groups of neighboring lecithin moieties. ${ }^{4,18,20}$ (Such a scenario can also explain why other hydrogen-bonding solvents have a similar effect as water on reverse micellar growth. ${ }^{18}$ ) We believe that bile salt molecules, due to their planar structure, are distributed in a similar fashion between the lecithin headgroups. This explains why water and bile salts have similar effects on micellar growth. Note that the volume of a bile salt molecule is much larger than that of water, and therefore, a few molecules of bile salt can have the same effect on headgroup size as a much larger number of water molecules. This aspect can help explain why a larger molar ratio of water compared to bile salt is necessary to reach the viscosity maximum; i.e., why $w_{0}{ }^{\max }$ is always larger than $B_{0}{ }^{\max }$ in Figure 7.

\section{Conclusions}

The addition of bile salt to lecithin organosols induces a transition from discrete spherical reverse micelles to entangled networks of wormlike reverse micelles. In turn, the zero-shear viscosity rises by more than 5 orders of magnitude, and the fluid shows a viscoelastic response with a single dominant relaxation time (Maxwell fluidlike behavior). SANS measurements further confirm the presence of flexible wormlike cylinders in these samples. Micellar growth has been demonstrated with four different bile salts, and in each case, the molar ratio of bile salt to lecithin is the controlling parameter. We attribute the micellar growth to a change in molecular geometry caused by the binding of bile salt molecules to lecithin headgroups (and the resulting expansion of the headgroup area). The unique "facially amphiphilic" structure of bile salts allow them to get sequestered between lecithin headgroups in a manner similar to water, which explains why bile salts and water have analogous effects on reverse micellar growth.

Acknowledgment. We would like to acknowledge NIST for facilitating the SANS experiments performed as part of this work. This work was partially funded by grants from NIST BFRL and Du Pont.

Supporting Information Available: A detailed Experimental Section is available. This section also includes a description of the SANS models used. This material is available free of charge via the Internet at http://pubs.acs.org.

\section{JA0583766}

(37) Schurtenberger, P.; Mazer, N.; Kanzig, W. J. Phys. Chem. 1985, 89, $1042-$ 1049

(38) Egelhaaf, S. U.; Schurtenberger, P. J. Phys. Chem. 1994, 98, 8560-8573. 


\title{
Supporting Information for
}

\section{A New Reverse Wormlike Micellar System: Mixtures of Bile Salt and Lecithin in Organic Liquids}

\author{
Shih-Huang Tung, Yi-En Huang and Srinivasa R. Raghavan* \\ Department of Chemical \& Biomolecular Engineering \\ University of Maryland \\ College Park, MD 20742-2111 \\ *Corresponding author. email: sraghava@eng.umd.edu
}

\section{EXPERIMENTAL SECTION}

Materials. Soybean lecithin (95\% purity) was purchased from Avanti Polar Lipids, Inc. The bile salts, sodium deoxycholate (SDC, > 97\% purity), sodium cholate (SC, > 99\%), sodium tauro-deoxycholate (STDC, > 97\%), and sodium taurocholate (STC, > 97\%) were purchased from Sigma-Aldrich. Cyclohexane, iso-octane, and isopropyl palmitate were purchased from EM Sciences, Fisher and TCI, respectively. n-hexane, 1-hexene, and n-decane were purchased from Sigma-Aldrich. Deuterated cyclohexane $(99.5 \%$ D) was obtained from Cambridge Isotopes.

Sample Preparation. Mixed solutions containing bile salt and lecithin were prepared as follows. Lecithin and bile salt were dissolved in methanol to form $200 \mathrm{mM}$ and $100 \mathrm{mM}$ stock solutions, respectively. Samples of desired composition were prepared by mixing the stock solutions. Methanol was removed by drying the samples in a vacuum oven at $50^{\circ} \mathrm{C}$ for 48 hours. The final samples with desired concentrations were obtained by adding the organic solvent, followed by stirring till the solutions became transparent and homogeneous. The above procedure ensured the removal of any residual water from the 
sample, and thereby facilitated reproducible sample preparation. Residual water content in the dried bile salt samples was studied using ${ }^{1} \mathrm{H}$ NMR on a Bruker $400 \mathrm{MHz}$ spectrometer. The studies revealed the presence of residual water at a 0.9:1 molar ratio in these samples. This aspect is further elaborated in the Discussion section of the paper.

Rheology. Steady and dynamic rheological experiments were performed on a Rheometrics RDA-III strain-controlled rheometer. A parallel-plate geometry of $25 \mathrm{~mm}$ radius was used for all samples. The plates were equipped with Peltier-based temperature control and all samples were studied at $25 \pm 0.1^{\circ} \mathrm{C}$. A solvent trap was used to minimize solvent evaporation. Frequency spectra were conducted in the linear viscoelastic regime of the samples, as determined from dynamic strain sweep measurements. For the steady shear experiments, sufficient time was allowed before data collection at each shear rate so as to ensure that the viscosity reached its steady-state value.

Small Angle Neutron Scattering (SANS). SANS measurements were made on the NG-7 (30 m) beamline at NIST in Gaithersburg, MD. Neutrons with a wavelength of $6 \AA$ were selected. Two sample-detector distances of $1.2 \mathrm{~m}$ and $15 \mathrm{~m}$ were used to probe a wide range of wave vectors from $0.004-0.4 \AA^{-1}$. Lecithin-bile salt samples were prepared with deuterated cyclohexane and studied in $1 \mathrm{~mm}$ quartz cells at $25^{\circ} \mathrm{C}$. The scattering spectra were corrected and placed on an absolute scale using calibration standards provided by NIST. The data are shown for the radially averaged intensity $I$ versus the wave vector $q=(4 \pi / \lambda) \sin (\theta / 2)$, where $\lambda$ is the wavelength of incident neutrons and $\theta$ is the scattering angle.

SANS Modeling. For dilute solutions of non-interacting scatterers, the SANS intensity $I(q)$ can be modeled purely in terms of the form factor $P(q)$ of the scatterers (i.e., the structure factor $S(q) \rightarrow 1$ in this case). In this study, we consider form factor models for three different micellar shapes: ellipsoids, rigid cylinders, and flexible cylinders. ${ }^{1,2}$ In the expressions below, $(\Delta \rho)$ is the difference in scattering length density between the micelle and the solvent, so that $(\Delta \rho)^{2}$ is the scattering contrast. The models were implemented using software modules supplied by NIST. 
Ellipsoids. The form factor $P(q)$ for ellipsoids of revolution with minor and major axes $R_{\mathrm{a}}$ and $R_{\mathrm{b}}$ is given by: ${ }^{1,2}$

$$
P(q)=(\Delta \rho)^{2}\left(\frac{4}{3} \pi R_{\mathrm{a}} R_{\mathrm{b}}^{2}\right)^{2} \int_{0}^{1}\left[3 \frac{(\sin z-z \cos z)}{z^{3}}\right]^{2} d \mu
$$

where $z=q \sqrt{\mu^{2} R_{\mathrm{b}}^{2}+R_{\mathrm{a}}^{2}\left(1-\mu^{2}\right)}$. Here $\mu$ is the cosine of the angle between the scattering vector $q$ and the symmetry axis of the ellipsoid.

Rigid Cylinders. The form factor $P(q)$ for rigid cylindrical rods of radius $R$ and length $L$ is given by: $:^{1,2}$

$$
P(q)=(\Delta \rho)^{2}\left(\pi R^{2} L\right)^{2} \int_{0}^{\pi / 2}[F(q, \alpha)]^{2} \sin \alpha d \alpha
$$

where $\quad F(q, \alpha)=\frac{J_{1}(q R \sin \alpha)}{(q R \sin \alpha)} \cdot \frac{\sin (q L \cos \alpha / 2)}{(q L \cos \alpha / 2)}$

here $\alpha$ is the angle between the cylinder axis and the scattering vector $q$ and $J_{1}(x)$ is the first-order Bessel function of the first kind.

Flexible Cylinders. The form factor for semiflexible chains of contour length $L$, persistence length $l_{p}$ and cross-sectional radius $R$ can be represented as the product of a cross-sectional form factor $P_{\mathrm{CS}}$ and wormlike-chain form factor $P_{\mathrm{Wc}}::^{2,3}$

$$
P(q)=P_{\mathrm{CS}}(q, R) \cdot P_{\mathrm{WC}}\left(q, L, l_{p}\right)
$$

The cross section form factor $P_{C S}$ can be approximated by the following expression, which is valid for cylindrical chains: ${ }^{2,3}$

$$
P_{\mathrm{CS}}(q, R)=\left[\frac{2 J_{1}(q R)}{q R}\right]^{2}
$$

The form factor $P_{\mathrm{WC}}$ for a wormlike chain with excluded-volume interactions is detailed in the paper by Pedersen and Schurtenberger, ${ }^{3}$ where it was originally derived. 


\section{REFERENCES}

[1] Feigin, L. A.; Svergun, D. I. Structure Analysis by Small-Angle X-Ray and Neutron Scattering; Plenum Press: New York, 1987.

[2] Pedersen, J. S. “Analysis of small-angle scattering data from colloids and polymer solutions: modeling and least-squares fitting.” Adv. Colloid Interface Sci. 1997, 70, 171210.

[3] Pedersen, J. S.; Schurtenberger, P. "Scattering functions of semiflexible polymers with and without excluded volume effects.” Macromolecules 1996, 29, 7602-7612. 